\title{
Editorial
}

\section{Quelle est la stratégie idéale pour traiter l'hyperglycémie du diabète de type 2 ?}

\section{Which ideal strategy for the management of hyperglycaemia in type 2 diabetes ?}

\author{
André J. Scheen \\ Professeur de Médecine, Université de Liège. Service de Diabétologie, Nutrition et Maladies \\ métaboliques et Unité de Pharmacologie clinique, \\ C.H.U. Sart Tilman, Liège, B-4000 Liège, Belgique. \\ Ancien Président de la Société Francophone du Diabète
}

Téléphone : 32-4-3667238

Télécopie : 32-4-3667068

Courriel : andre.scheen@chu.ulg.ac.be

Le diabète de type 2 (DT2) est la forme de diabète sucré de loin la plus fréquente et ce, dans tous les pays du monde. Il ne fait aucun doute, cependant, que ce concept de DT2 correspond à une entité hétérogène et que cette dernière sera démembrée dans un avenir sans doute relativement proche, compte tenu des progrès considérables de la biologie moléculaire ${ }^{(1)}$. Par ailleurs, les thérapeutiques proposées sont de plus en plus nombreuses, complexes et onéreuses ${ }^{(2)}$, de telle sorte qu'il n’est pas aisé, pour le praticien, de choisir la meilleure approche thérapeutique. Ainsi, les recommandations internationales sont de plus en plus sophistiquées ${ }^{(3)}{ }^{(4)}$ et peuvent dérouter le non spécialiste confronté à une prise de décision individuelle face à un patient déterminé.

Ce qu'il est convenu d'appeler le DT2 présente deux caractéristiques essentielles : d'une part, il s'agit d'une maladie à la physiopathologie complexe, impliquant de nombreux 
déficits touchant plusieurs organes (pancréas, foie, muscle, tissu adipeux, intestin, rein) ${ }^{(5)}$; d'autre part, il s'agit d’une maladie évolutive au cours du temps chez un même individu, notamment en raison d'un effondrement progressif de la fonction (et de la masse) des cellules B pancréatiques ${ }^{(6)}$. Ces deux caractéristiques ont des conséquences pratiques évidentes en ce qui concerne les stratégies thérapeutiques ciblant l’hyperglycémie.

Au vu de la complexité du DT2 impliquant de multiples déficits, il est sans doute illusoire qu'un seul médicament puisse corriger toutes les anomalies ${ }^{(5)}$. Ceci a pour conséquence qu’une combinaison de plusieurs médicaments est le plus souvent nécessaire pour maîtriser l’hyperglycémie de façon durable. Plutôt que d'adopter la stratégie étape par étape, commençant par la metformine puis ajoutant progressivement d'autres médicaments en cas d'échec et de non obtention de la cible jusqu’à, finalement, un certain empilement, comme proposé dans la plupart des recommandations internationales récentes ${ }^{(3)}{ }^{(4)}$, certains préconisent une stratégie plus agressive d’emblée (Figure 1$)^{(7)}$. Celle-ci consiste, dès le diagnostic de DT2, en la combinaison de plusieurs médicaments à effets complémentaires de façon à cibler les différents mécanismes physiopathologiques, à mieux contrôler l'hyperglycémie et, surtout, à assurer une meilleure durabilité de l'action antihyperglycémiante, garante d'une réduction des complications à terme ${ }^{(6)}$. Cependant, on ne peut s’empêcher de penser que nombre de patients soumis à ce traitement intensif d'emblée n'en ont peut-être pas besoin, en raison d'une maladie moins agressive. Si tel est le cas, ces patients sont exposés inutilement à des médicaments dont certains peuvent éventuellement engendrer des manifestations indésirables. Par ailleurs, il est évident qu’une telle stratégie combinant d'emblée plusieurs médicaments augmente sensiblement le coût des traitements pharmacologiques, par comparaison à un traitement intensifié par étape selon les besoins.

Au vu de l'évolutivité du DT2, il est indispensable d'ajuster progressivement le traitement au cours du temps, soit en augmentant les posologies, soit en recourant à des combinaisons thérapeutiques, si possible à actions complémentaires (Figure 1$)^{(3)}{ }^{(4)}$. Cette stratégie ne se différencierait pas fondamentalement de la stratégie précédente, si, en pratique, on n’était pas confronter à ce qui est couramment appelé une inertie clinique, phénomène bien connu dans la prise en charge d'une maladie chronique asymptomatique comme le DT2 ${ }^{\text {(8) (9) }}$. Celle-ci aboutit à un retard, parfois considérable, dans l'intensification du traitement antihyperglycémiant alors que la thérapeutique mise en œuvre s’avère, manifestement, insuffisante. Vaincre cette inertie thérapeutique repose sur plusieurs éléments ${ }^{(8)(9)}$ : tout d'abord, être convaincu du bien-fondé de l'intensification du traitement, ensuite disposer de 
médications efficaces et sûres pour la réaliser, enfin maîtriser les modalités, pour ne pas dire les subtilités, d’une stratégie optimale d'intensification du traitement ${ }^{(3)}{ }^{(4)}$.

Quelle que soit la stratégie privilégiée (une polymédication d’emblée ou une intensification rapidement progressive du traitement pharmacologique), le succès dépend d'un élément crucial, à savoir la bonne adhésion du patient au traitement proposé, concept connu sous le nom d'observance thérapeutique ${ }^{(10)}$. En effet, dans les deux cas de figure, le patient DT2 est, tôt ou tard, confronté avec la prise de plusieurs médicaments anti-hyperglycémiants. Ceux-ci doivent, dans la plupart des cas, être associés à d’autres médications visant à réduire le risque cardiovasculaire élevé, à savoir un ou, plus souvent, plusieurs antihypertenseurs, un hypolipidémiant et, éventuellement, un antiagrégant plaquettaire. Devant une maladie le plus souvent asymptomatique, il n'est guère étonnant que l’observance thérapeutique soit largement imparfaite chez bon nombre de patients DT2. Ce manque d'adhésion est sans doute plus marqué dans les conditions de vie réelle que dans les conditions artificielles des essais cliniques contrôlés réalisés chez des patients sélectionnés et particulièrement bien surveillés ${ }^{(11)}$. Limiter le phénomène de la mauvaise adhésion au traitement représente un défi majeur, reconnu par l'Organisation Mondiale de la Santé pour toutes les maladies chroniques dont le DT2 ${ }^{(12)}$. Le développement de nouvelles approches pharmacologiques, mieux tolérées (moins ou pas d'hypoglycémies, absence de prise de poids) ou plus faciles à prendre (prise hebdomadaire, par exemple) est susceptible de contribuer à améliorer cette problématique de mauvaise adhésion au traitement. Cependant, un autre frein pourrait être d'ordre économique, surtout dans les pays moins favorisés. En effet, les médicaments modernes qui offrent une série d'avantages sont également plus onéreux ${ }^{(2)}$, ce qui peut en limiter l’accessibilité, soit au niveau de la collectivité, soit au niveau individuel, mais aussi l'observance pour des raisons de coût.

Compte tenu de l’hétérogénéité probable du DT2 ${ }^{(1)}$, il est sans doute illusoire que la même approche thérapeutique, plus ou moins standardisée, intensive d’emblée ou rapidement progressive, soit applicable avec la même efficacité chez tous les patients atteints de cette maladie. Il est connu que les réponses peuvent être très variables, avec de bons répondeurs et de mauvais répondeurs. Une approche centrée sur le patient et une approche algorithmique standardisée sont sans doute quelque peu antinomiques ${ }^{(13)}$. Dès lors, plutôt que d'empiler des médicaments plus ou moins efficaces, il serait sans doute plus judicieux de sélectionner le bon antidiabétique pour le bon patient au bon moment (« the right drug for the right patient at the right time »). Jusqu’à présent, les choix préférentiels se fondent sur des critères 
phénotypiques, cliniques (patient obèse ou non, par exemple) ou biologique (peptide-C effondré ou restant élevé, niveau d’élévation du taux d’hémoglobine glyquée, par exemple) (14). Ces critères s’avèrent, cependant, largement imparfaits. Ceci ouvre la voie à la médecine personnalisée qui fera, tôt ou tard, appel à une approche génotypique ${ }^{(15)}$. On pourrait rêver d’un traitement simplifié, sélectionnant la molécule la plus efficace pour un patient donné, en fonction de ses caractéristiques génétiques propres, ce qui permettrait d’éviter de devoir recourir à l'empilement des molécules souvent utilisé actuellement (Figure 1). Cette approche, sans doute inéluctable à terme, au vu des énormes progrès de la biologie moléculaire et de la diminution des coûts du génotypage des individus, dictera une nouvelle médecine. Il restera à démontrer qu’elle s’avérera plus performante que les stratégies utilisées actuellement et que sa rentabilité pharmaco-économique sera compétitive vis-à-vis des pratiques plus communément utilisées actuellement. Certes, celles-ci ont leurs limites, mais elles peuvent s’avérer néanmoins remarquablement performantes si elles sont bien maîtrisées et correctement appliquées sur le terrain ${ }^{(13)}$.

Figure 1 : Illustration schématique de trois stratégies de prise en charge de l’hyperglycémie dans le diabète de type 2. Gauche : Empilement progressif des médications antihyperglycémiantes en fonction de la progression de la maladie. Milieu : Traitement agressif d'emblée faisant appel à une combinaison de traitements à actions complémentaires. Droite : Médecine personnalisée du futur, sélectionnant la bonne molécule dotée d’une excellente réponse prolongée et ce, pour chaque patient individuel.

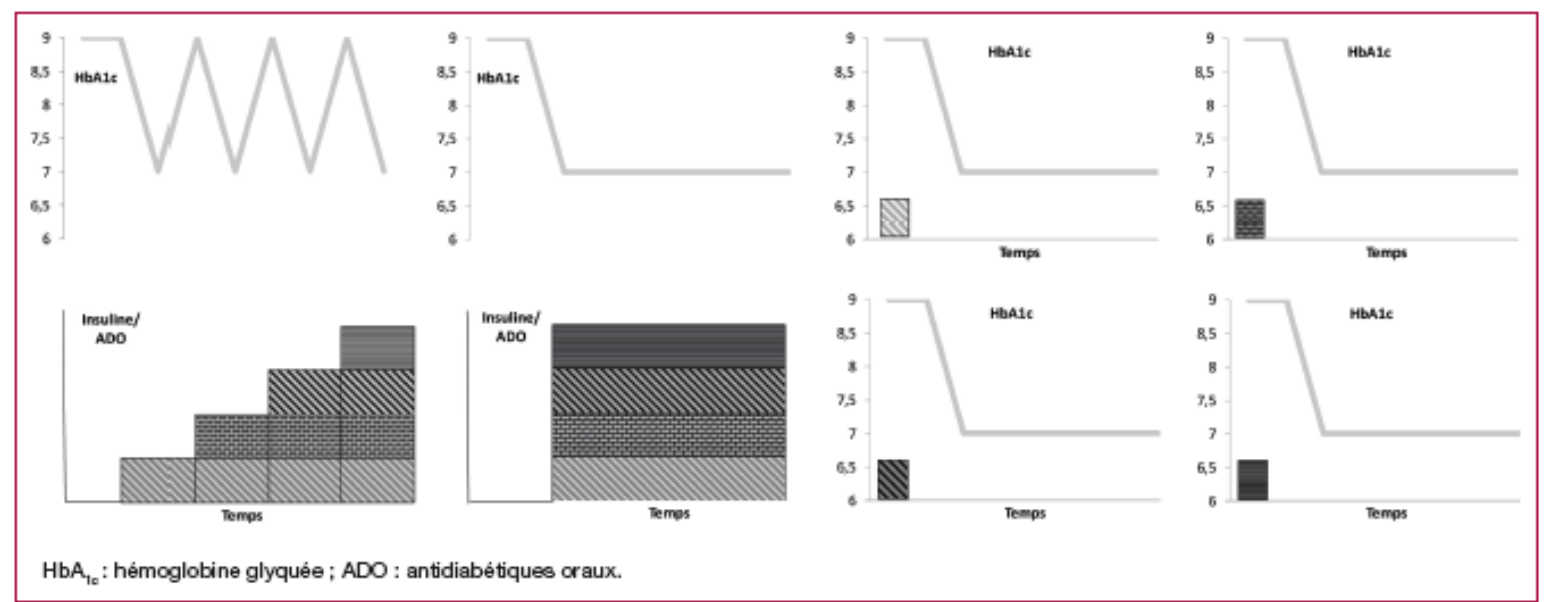

Figure 1. Illustration schématique de trois stratégies de prise en charge de Phyperglycémie dans le diabète de type 2. Gauche : Empilement progressif des médications anti-hyperglycémiantes en fonction de la progression de la maladie.

Milieu : Traitement agressif d'emblée faisant appel à une combinaison de traitements à actions complémentaires.

Droite : Médecine personnalisée du futur, sélectionnant la bonne molécule dotée d'une excellente réponse prolongée et ce, pour chaque patient individuel. 


\section{Déclaration d'intérêt}

L’auteur déclare n’avoir aucun conflit d’intérêt en lien direct avec ce manuscrit. Il a reçu des honoraires, à titre personnel ou institutionnel, comme orateur, conseiller scientifique et/ou investigateur clinicien de la part des firmes suivantes : AstraZeneca/BMS, Boehringer Ingelheim, Eli Lilly, GlaxoSmithKline, Janssen, Merck Sharp \& Dohme, Novartis, NovoNordisk, Sanofi and Takeda.

\section{Bibliographie}

[1] Faerch K, Hulman A, Solomon TP. Heterogeneity of pre-diabetes and type 2 diabetes: implications for prediction, prevention and treatment responsiveness. Curr Diabetes Rev 2016;12(1):30-41.

[2] Scheen AJ. Antidiabétiques oraux dans le traitement du diabète de type 2 : perspectives historique et médico-économique Médecine des maladies Métaboliques 2015;9:186-97. [3] Inzucchi SE, Bergenstal RM, Buse JB, et al. Management of hyperglycaemia in type 2 diabetes, 2015: a patient-centred approach. Update to a position statement of the American Diabetes Association and the European Association for the Study of Diabetes. Diabetologia 2015;58(3):429-42.

[4] Garber AJ, Abrahamson MJ, Barzilay JI, et al. Consensus Statement by the American Association of Clinical Endocrinologists and American College of Endocrinology on the Comprehensive Type 2 Diabetes Management Algorithm - 2016 Executive Summary. Endocr Pract 2016;22(1):84-113.

[5] DeFronzo RA. Banting Lecture. From the triumvirate to the ominous octet: a new paradigm for the treatment of type 2 diabetes mellitus. Diabetes 2009;58(4):773-95. [6] Bailey CJ, Del Prato S, Eddy D, et al. Earlier intervention in type 2 diabetes: the case for achieving early and sustained glycaemic control. Int J Clin Pract 2005;59(11):1309-16. [7] Abdul-Ghani MA, Puckett C, Triplitt C, et al. Initial combination therapy with metformin, pioglitazone and exenatide is more effective than sequential add-on therapy in subjects with new-onset diabetes. Results from the Efficacy and Durability of Initial Combination Therapy for Type 2 Diabetes (EDICT): a randomized trial. Diabetes Obes Metab 2015;17(3):268-75. [8] Scheen AJ. Inertie thérapeutique dans la pratique médicale: causes, conséquences, solutions. Rev Med Liège 2010;65(5-6):232-8. 
[9] Reach G. Clinical inertia, uncertainty and individualized guidelines. Diabetes Metab 2014;40(4):241-5.

[10] Scheen AJ, Giet D. Non-observance thérapeutique : causes, conséquences, solutions. Rev Med Liège 2010;65(5-6):232-8.

[11] Strain WD, Bluher M, Paldanius P. Clinical inertia in individualising care for diabetes: is there time to do more in type 2 diabetes? Diabetes Ther 2014;5(2):347-54.

[12] Worl Health Organization. Adherence to long-term therapies. Evidence for action. http://wwwwhoint/chp/knowledge/publications/adherence_full_reportpdf 2003.

[13] Raz I, Riddle MC, Rosenstock J, et al. Personalized management of hyperglycemia in type 2 diabetes: reflections from a Diabetes Care Editors' Expert Forum. Diabetes Care 2013;36(6):1779-88.

[14] Ceriello A, Gallo M, Candido R, et al. Personalized therapy algorithms for type 2 diabetes: a phenotype-based approach. Pharmgenomics Pers Med 2014;7:129-36. [15] Scheen AJ. Personalized medicine in type 2 diabetes. Diabetes Res Clin Pract 2016:Submitted. 